\title{
Deaths due to COVID-19 in Affected Countries
}

\author{
Piryani Rano $\mathrm{Mal}^{1 *}$, Piryani Suneel ${ }^{2}$, Bhandary Shital ${ }^{3}$ \\ Department of Internal Medicine, Universal College of Medical Sciences, Bhairahawa, Nepal \\ ${ }^{2}$ Department of Community Health Sciences, Aga Khan University, Karachi, Pakistan \\ ${ }^{3}$ School of Public Health, Patan Academy of Health Sciences, Lalitpur, Nepal
}

Article Info

\section{Article Notes}

Received: April 6, 2020

Accepted: April 13, 2020

\section{${ }^{*}$ Correspondence:}

Rano Mal Piryani, Professor of Internal Medicine and Head of Department of Internal Medicine, Chief Coordinator-Health Professions Training Committee, Universal College of Medical Sciences, Bhairahawa, Nepal; Telephone No: 00977-9841269522; Email: rano.piryani@gmail.com, r_piryani@hotmail.com.

c 2020 Piryani RM. This article is distributed under the terms of the Creative Commons Attribution 4.0 International License.
A new coronavirus called as novel coronavirus was first emerged in Wuhan, Hubei province of China, at the end of 2019. This was labelled as 2019-nCoV. A first patient of pneumonia of unknown cause was detected in Wuhan, China. The case was reported to the World Health Organization (WHO) Country Office in China on 31 December 2019. Subsequently, the outbreak was declared a Public Health Emergency of International Concern on January 30, 2020. WHO announced a name for the new coronavirus disease, COVID-19 on February 11, 2020. When COVID-19 infection reported from 114 countries then WHO declared COVID-19 infection, a global pandemic on March 11, 2020. Till the writing of this article April 2, 2020, the COVID-19 affected 200 countries and territories around the world and 2 international conveyance (the Diamond Princess cruise ship harbored in Yokohama, Japan and the Holland America's MS Zaandam cruise ship) ${ }^{1,2,3,4}$.

Initially, the epicenter was China, later on it was shifted to Italy, then Spain and now epicenter is United States of America. Deaths reported from various countries are varied relative to total new cases reported. The pandemic is very rapidly accelerating. The first case was reported on Dec 31, 2019; 1st one lac cases in 67 days, 2nd in 11 days, 3 rd in 4 days, 4 th in 3 days, 5 th in 2.5 days, 6 th in 2 days and $7^{\text {th }} 2$ days and 8 th 1.5 days and $9^{\text {th }}$ in one day ${ }^{5}$.

We retrieved the secondary data of countries those reported more than 1000 new cases of COVID-19 until 13.36 Greenwich Mean Time March 30, 2020 from worldmeter website. https:// www.worldometers.info/coronavirus/. (Table 1). Forty-two countries reported more than 1000 cases. In 18 countries first case was reported in January, 17 countries reported their first case in February while 7 in March ${ }^{4}$. The data related to total new cases and total deaths were entered into IBM Statistical Package for the Social Sciences version 21 and percentage of death was calculated. The highest number of cases reported from United States of America 142,793 while lowest from Iceland 1,020 among 42 countries. The maximum deaths were reported from Italy 10,779 while lowest from South Africa and Iceland i.e. 2 from each country. (Table 1, Figure 1 and 2) The percentage of deaths was highest among patients of Italy while lowest in Iceland among 42 countries.

There is variation observed in percentage of deaths among 42 countries. Why this is $\mathrm{so}^{6,7,8}$ ?

The possible and probable reasons for this variation could be: in population affected in countries; in people with comorbidities in different countries; in lab test done in countries; in strategies adopted 
Table 1. showing Total cases, New Cases, Total Deaths, New Deaths, Total Recovered, Active Cases, Serious Critical Total Case/1MP, Death/1MP, Reported 1 Cases.

\begin{tabular}{|c|c|c|c|c|c|c|c|c|c|c|}
\hline \multicolumn{11}{|c|}{$\begin{array}{l}\text { COVID-19 CORONAVIRUS PANDEMIC } \\
\text { Last updated: March 30, 2020, 13:36 GMT }\end{array}$} \\
\hline World & 739,371 & $+17,012$ & 35,016 & $+1,050$ & 156,402 & 547,953 & 28,434 & 94.9 & 4.5 & Jan 10 \\
\hline $\begin{array}{l}\text { Country } \\
\text { other }\end{array}$ & $\begin{array}{l}\text { Total } \\
\text { cases }\end{array}$ & $\begin{array}{l}\text { New } \\
\text { cases }\end{array}$ & $\begin{array}{l}\text { Total } \\
\text { Deaths }\end{array}$ & $\begin{array}{l}\text { New } \\
\text { Deaths }\end{array}$ & $\begin{array}{c}\text { Total } \\
\text { Recovered }\end{array}$ & $\begin{array}{l}\text { Active } \\
\text { Cases }\end{array}$ & $\begin{array}{l}\text { Serious } \\
\text { Critical }\end{array}$ & $\begin{array}{c}\text { Total } \\
\text { case /1MP }\end{array}$ & Death/1MP & $\begin{array}{l}\text { Reported } \\
\text { 1sr case }\end{array}$ \\
\hline USA & 142,793 & +333 & 2,490 & +6 & 4,562 & 135,741 & 2,970 & 431 & 8 & Jan 20 \\
\hline Italy & 97,689 & & 10,779 & & 13,030 & 73,880 & 3,906 & 1,616 & 178 & $\operatorname{Jan} 29$ \\
\hline Spain & 85,195 & $+5,085$ & 7,340 & +537 & 16,780 & 61,075 & 5,231 & 1,822 & 157 & Jan 30 \\
\hline China & 81,470 & +31 & 3,304 & +4 & 75,700 & 2,466 & 633 & 57 & 2 & $\operatorname{Jan} 10$ \\
\hline Germany & 63,079 & +644 & 545 & +4 & 9,211 & 53,323 & 1,979 & 753 & 7 & $\operatorname{Jan} 26$ \\
\hline Iran & 41,495 & $+3,186$ & 2,757 & +117 & 13,911 & 24,827 & 3,511 & 494 & 33 & Feb 18 \\
\hline France & 40,174 & & 2,606 & & 7,202 & 30,366 & 4,632 & 615 & 40 & $\operatorname{Jan} 23$ \\
\hline$\underline{\mathrm{UK}}$ & 19,522 & & 1,228 & & 135 & 18,159 & 163 & 288 & 18 & $\operatorname{Jan} 30$ \\
\hline Switzerland & 15,526 & +697 & 333 & +33 & 1,823 & 13,370 & 301 & 1,794 & 38 & Feb 24 \\
\hline Belgium & 11,899 & $+1,063$ & 513 & +82 & 1,527 & 9,859 & 927 & 1,027 & 44 & Feb 03 \\
\hline Netherlands & 11,750 & +884 & 864 & +93 & 250 & 10,636 & 1,053 & 686 & 50 & Feb 26 \\
\hline S. Korea & 9,661 & +78 & 158 & +6 & 5,228 & 4,275 & 59 & 188 & 3 & Jan 19 \\
\hline Austria & 9,377 & +589 & 108 & +22 & 636 & 8,633 & 193 & 1,041 & 12 & Feb 24 \\
\hline Turkey & 9,217 & & 131 & & 105 & 8,981 & 568 & 109 & 2 & Mar 09 \\
\hline Portugal & 6,408 & +446 & 140 & +21 & 43 & 6,225 & 164 & 628 & 14 & Mar 01 \\
\hline Canada & 6,320 & & 65 & & 573 & 5,682 & 120 & 167 & 2 & Jan 24 \\
\hline Norway & 4,393 & +109 & 31 & +5 & 12 & 4,350 & 97 & 810 & 6 & Feb 25 \\
\hline Israel & 4,347 & +100 & 16 & +1 & 134 & 4,197 & 80 & 502 & 2 & Feb 20 \\
\hline Brazil & 4,316 & +60 & 139 & +3 & 120 & 4,057 & 296 & 20 & 0.7 & Feb 24 \\
\hline Australia & 4,245 & +82 & 18 & +1 & 244 & 3,983 & 28 & 166 & 0.7 & $\operatorname{Jan} 24$ \\
\hline Sweden & 4,028 & +328 & 146 & +36 & 16 & 3,866 & 306 & 399 & 14 & $\operatorname{Jan} 30$ \\
\hline Czechia & 2,878 & +61 & 17 & +1 & 11 & 2,850 & 52 & 269 & 2 & Feb 29 \\
\hline Malaysia & 2,626 & +156 & 37 & +2 & 479 & 2,110 & 94 & 81 & 1 & $\operatorname{Jan} 24$ \\
\hline Ireland & 2,615 & & 46 & & 5 & 2,564 & 59 & 530 & 9 & Feb 28 \\
\hline Denmark & 2,555 & +160 & 77 & +5 & 1 & 2,477 & 113 & 441 & 13 & Feb 26 \\
\hline Chile & 2,449 & +310 & 8 & +1 & 156 & 2,285 & 14 & 128 & 0.4 & Mar 02 \\
\hline Luxembourg & 1,988 & +38 & 22 & +1 & 40 & 1,926 & 25 & 3,176 & 35 & Feb 28 \\
\hline Poland & 1,984 & +122 & 26 & +4 & 7 & 1,951 & 3 & 52 & 0.7 & Mar 03 \\
\hline Romania & 1,952 & +137 & 46 & +3 & 206 & 1,700 & 31 & 101 & 2 & Feb 25 \\
\hline Ecuador & 1,924 & & 58 & & 3 & 1,863 & 58 & 109 & 3 & Feb 28 \\
\hline Japan & 1,866 & & 54 & & 424 & 1,388 & 56 & 15 & 0.4 & Jan 14 \\
\hline Russia & 1,836 & +302 & 9 & +1 & 66 & 1,761 & 8 & 13 & 0.06 & $\operatorname{Jan} 30$ \\
\hline Pakistan & 1,650 & +53 & 20 & +6 & 29 & 1,601 & 11 & 7 & 0.09 & Feb 25 \\
\hline Philippines & 1,546 & +128 & 78 & +7 & 42 & 1,426 & 1 & 14 & 0.7 & $\operatorname{Jan} 29$ \\
\hline Thailand & 1,524 & +136 & 9 & +2 & 229 & 1,286 & 11 & 22 & 0.1 & $\operatorname{Jan} 12$ \\
\hline Saudi Arabia & 1,453 & +154 & 8 & & 66 & 1,379 & 12 & 42 & 0.2 & Mar 01 \\
\hline Indonesia & 1,414 & +129 & 122 & +8 & 75 & 1,217 & & 5 & 0.4 & Mar 01 \\
\hline Finland & 1,352 & +112 & 13 & +2 & 10 & 1,329 & 49 & 244 & 2 & $\operatorname{Jan} 28$ \\
\hline South Africa & 1,280 & & 2 & & 31 & 1,247 & 7 & 22 & 0.03 & Mar 04 \\
\hline Greece & 1,156 & & 39 & & 52 & 1,065 & 66 & 111 & 4 & Feb 25 \\
\hline India & 1,071 & +47 & 29 & +2 & 100 & 942 & & 0.8 & 0.02 & $\operatorname{Jan} 29$ \\
\hline Iceland & 1,020 & & 2 & & 135 & 883 & 25 & 2,989 & 6 & Feb 27 \\
\hline
\end{tabular}

Source: COVID-19 CORONAVIRUS PANDEMIC. https://www.worldometers.info/coronavirus/ 


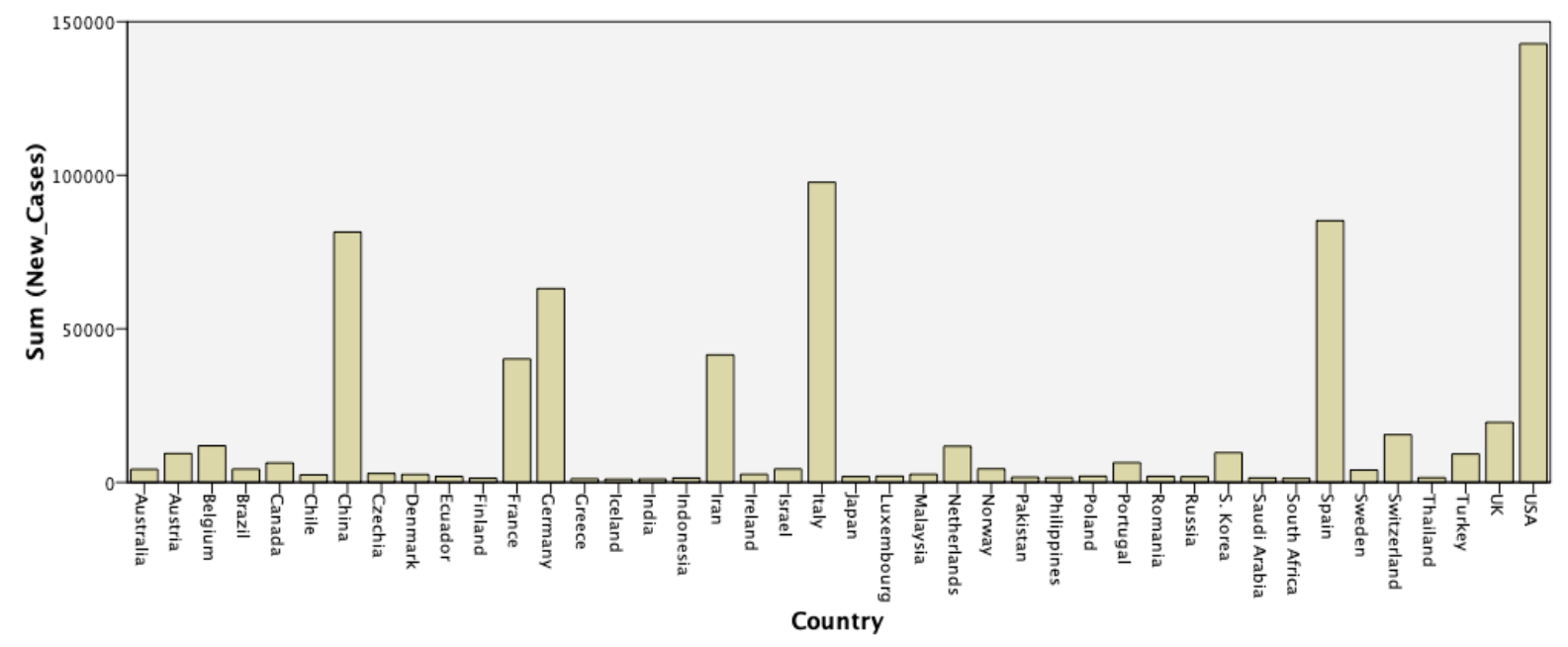

Source: COVID-19 CORONAVIRUS PANDEMIC. https://www.worldometers.info/coronavirus/

Figure 1: Total cases in 42 countries reported more than 1000 COVID-19 cases on March 30, 2020

Table 2. Total cases, total deaths and percentage of deaths in 42 countries reported more than 1000 Covid-19 cases on March 30,2020

\begin{tabular}{|l|c|c|c|c|c|c|c|c|c|c|c|}
\hline \multicolumn{1}{|c|}{ Country } & $\begin{array}{c}\text { Total New } \\
\text { Cases }\end{array}$ & $\begin{array}{c}\text { Total } \\
\text { Deaths }\end{array}$ & $\begin{array}{c}\text { Percentage } \\
\text { of Deaths }\end{array}$ & Country & $\begin{array}{c}\text { Total New } \\
\text { Cases }\end{array}$ & $\begin{array}{c}\text { Total } \\
\text { Deaths }\end{array}$ & $\begin{array}{c}\text { Percentage } \\
\text { of Deaths }\end{array}$ & Country & $\begin{array}{c}\text { Total New } \\
\text { Cases }\end{array}$ & $\begin{array}{c}\text { Total } \\
\text { Deaths }\end{array}$ & $\begin{array}{c}\text { Percentage } \\
\text { of Deaths }\end{array}$ \\
\hline USA & 142793 & 2490 & 1.74 & Portugal & 6408 & 140 & 2.18 & Romania & 1952 & 46 & 2.35 \\
\hline Italy & 97689 & 10779 & 11.03 & Canada & 6320 & 65 & 1.02 & Ecuador & 1924 & 58 & 3.01 \\
\hline Spain & 85195 & 7340 & 8.61 & Norway & 4393 & 31 & .70 & Japan & 1866 & 54 & 2.89 \\
\hline China & 81470 & 3304 & 4.00 & Israel & 4347 & 16 & .36 & Russia & 1836 & 9 & .49 \\
\hline Germany & 63079 & 545 & .86 & Brazil & 4316 & 139 & 3.22 & Pakistan & 1650 & 20 & 1.21 \\
\hline Iran & 41495 & 2757 & 6.64 & Australia & 4245 & 18 & .42 & Philippines & 1546 & 78 & 5.04 \\
\hline France & 40174 & 2606 & 6.48 & Sweden & 4028 & 146 & 3.62 & Thailand & 1524 & 9 & .59 \\
\hline UK & 19522 & 1228 & 6.29 & Czechia & 2878 & 17 & .59 & Saudi Arabia & 1453 & 8 \\
\hline Switzerland & 15526 & 333 & 2.14 & Malaysia & 2626 & 37 & 1.40 & Indonesia & 1414 & 122 & .55 \\
\hline Belgium & 11899 & 513 & 4.31 & Ireland & 2615 & 46 & 1.75 & Finland & 1352 & 13 & .96 \\
\hline Netherlands & 11750 & 864 & 7.35 & Denmark & 2555 & 77 & 3.01 & South Africa & 1280 & 2 \\
\hline S. Korea & 9661 & 158 & 1.63 & Chile & 2449 & 8 & .32 & Greece & 1156 & 39 \\
\hline Austria & 9377 & 108 & 1.51 & Luxembourg & 1988 & 22 & 1.10 & India & 1071 & 29 & 2.37 \\
\hline Turkey & 9217 & 131 & 1.42 & Poland & 1984 & 26 & 1.31 & Iceland & 1020 & 2 & .19 \\
\hline
\end{tabular}

Source: COVID-19 CORONAVIRUS PANDEMIC. https://www.worldometers.info/coronavirus/

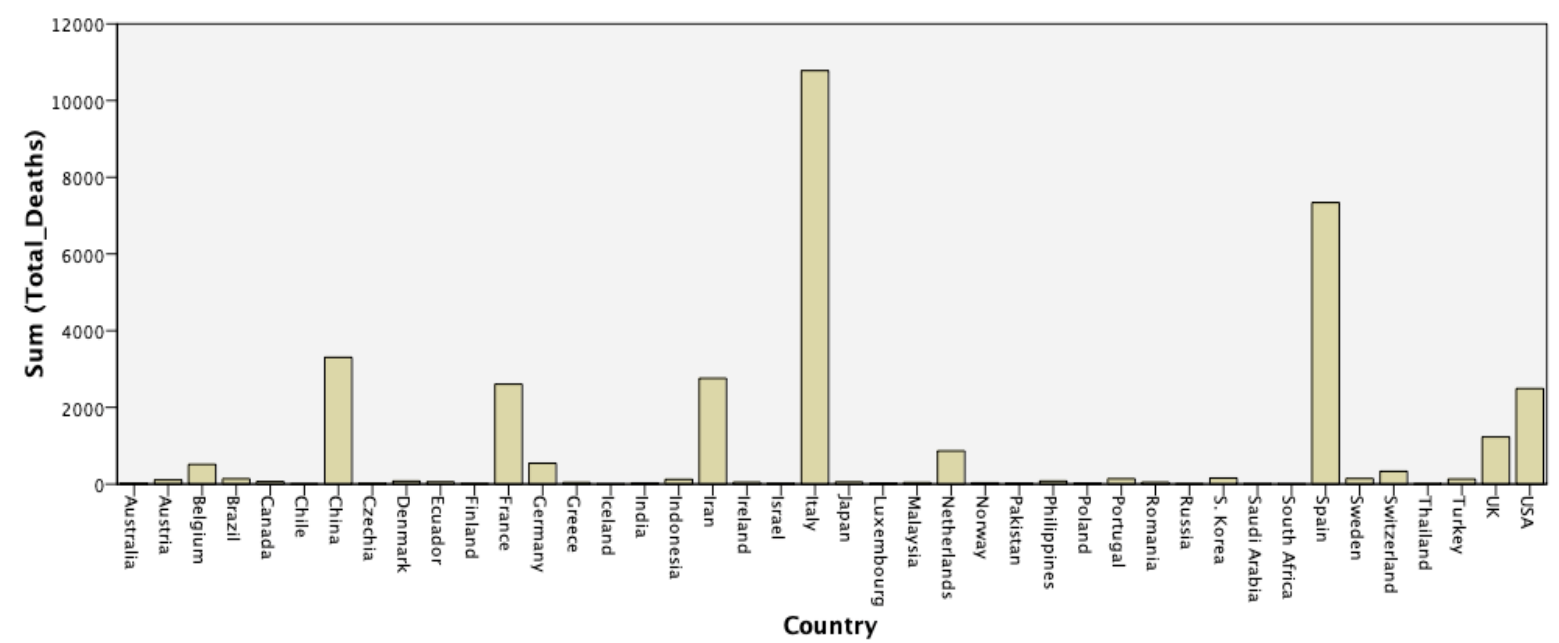

Source: COVID-19 CORONAVIRUS PANDEMIC. https://www.worldometers.info/coronavirus/

Figure 2: Total Deaths in 46 countries reported more than 1000 COVID-19 cases on March 30, 2020. 


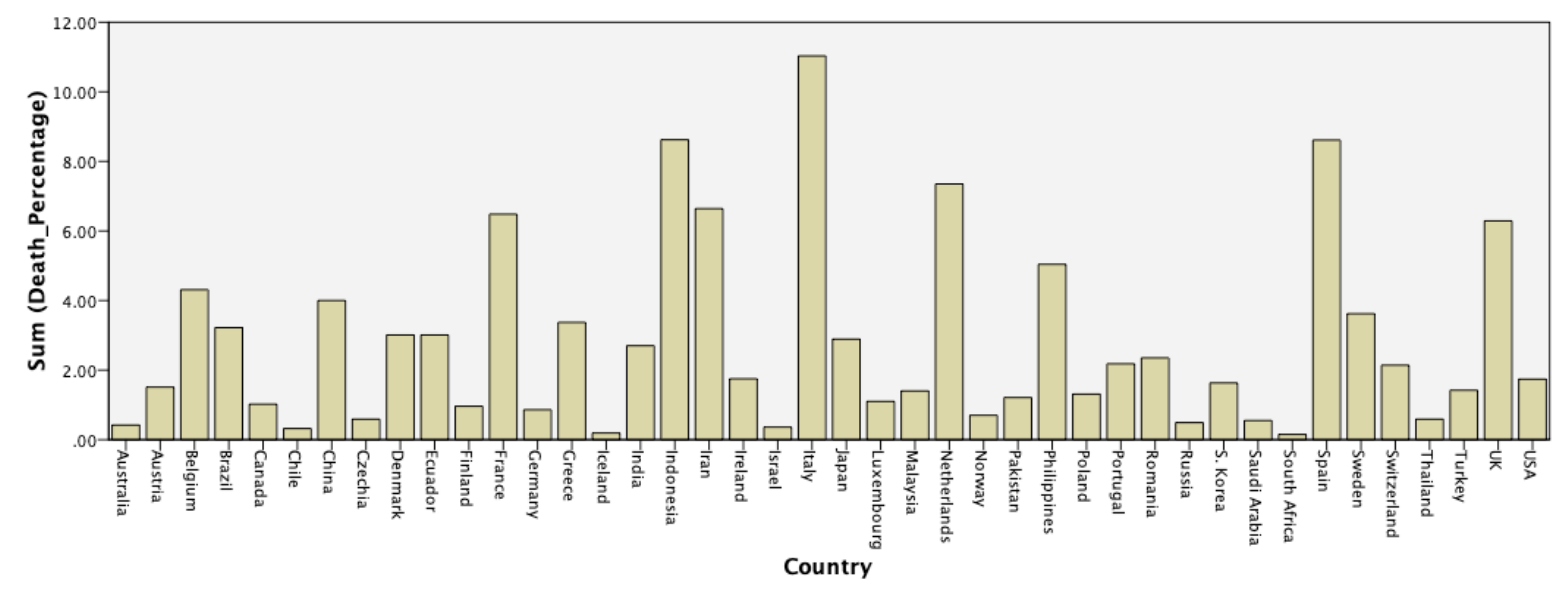

Source: COVID-19 CORONAVIRUS PANDEMIC. https://www.worldometers.info/coronavirus/

Figure 3: Percentage of Deaths in 42 countries reported more than 1000 COVID-19 cases on March 30, 2020.

for isolation and quarantine; in treatment especially drugs used and their adverse effects; in healthcare responses; in temperature between different countries, regions or in seasons; in preventive methods, their timing and frequency; in virulence of virus; in immunity differences among people of different countries or among different sections of people within same country; in awareness campaigns and public's response to it and possibilities of mutation of virus

It is recommended to know the exact reasons for this variation in COVID-19 deaths for future course of action. Hence, analytical studies or systemic review or metaanalysis are required to address this issue.

\section{References}

1. World Health Organisation. Global Tuberculosis Report 2019 [Internet]. Geneva, Switzerland; 2019. Available from: https://apps. who.int/iris/bitstream/handle/10665/329368/9789241565714eng.pdf?ua $=1$

2. Ryan H, Yoo J, Darsini P. Corticosteroids for tuberculous pleurisy. Cochrane database Syst Rev [Internet]. 2017 [cited 2019 Oct 6]; 3(3): CD001876. Available from: http://www.ncbi.nlm.nih.gov/ pubmed/28290161

3. Zhai K, Lu Y, Shi HZ. Tuberculous pleural effusion. J Thorac Dis
[Internet]. 2016 Jul [cited 2019 Oct 6]; 8(7): E486-94. Available from: http://www.ncbi.nlm.nih.gov/pubmed/27499981

4. Shaw JA, Irusen EM, Diacon AH, et al. Pleural tuberculosis: A concise clinical review. Clin Respir J. 2018 May 1; 12(5): 1779-86.

5. Cohen LA, Light RW. Tuberculous Pleural Effusion. Turkish Thorac J [Internet]. 2015 Jan [cited 2019 Oct 5]; 16(1): 1-9. Available from: http://www.ncbi.nlm.nih.gov/pubmed/29404070

6. Central TB Division (Ministry of Health and Family Welfare). India TB Report 2019: Revised National Tuberculosis Control Programme Annual Report [Internet]. Delhi, India; 2019. Available from: https:// tbcindia.gov.in/WriteReadData/India TB Report 2019.pdf

7. Sharma SK, Ryan H, Khaparde S, et al. Index-TB guidelines: Guidelines on extrapulmonary tuberculosis for India. Indian J Med Res [Internet]. 2017 Apr [cited 2019 Oct 6]; 145(4): 448-63. Available from: http:// www.ncbi.nlm.nih.gov/pubmed/28862176

8. Desai C, Shah A, Dikshit R, et al. Efficacy and safety of anti-tuberculosis drugs in HIV-positive patients: A prospective study. Indian J Pharmacol [Internet]. 2013 [cited 2019 Dec 10]; 45(5): 447. Available from: http://www.ncbi.nlm.nih.gov/pubmed/24130377

9. Wyser C, Walzl G, Smedema JP, et al. Corticosteroids in the treatment of tuberculous pleurisy: A double- blind, placebo-controlled, randomized study. Chest. 1996; 110(2): 333-8.

10. Antonangelo L, Faria CS, Sales RK. Tuberculous pleural effusion: diagnosis \& management. Expert Rev Respir Med [Internet]. 2019 Aug 3; 13(8): 747-59. Available from: https://doi.org/10.1080/174 76348.2019.1637737 